\title{
Aerospace Electromagnetic Thruster with Average Foucault Currents
}

\author{
Sergey A. Gerasimov \\ Southern Federal University, Rostov-on-Don, Russian Federation \\ Email: gsim1953@mail.ru
}

How to cite this paper: Gerasimov, S.A. (2022) Aerospace Electromagnetic Thruster with Average Foucault Currents. Advances in Aerospace Science and Technology, 7, 2531.

https://doi.org/10.4236/aast.2022.71002

Received: January 27, 2022

Accepted: March 1, 2022

Published: March 4, 2022

Copyright $\odot 2022$ by author(s) and Scientific Research Publishing Inc. This work is licensed under the Creative Commons Attribution International License (CC BY 4.0).

http://creativecommons.org/licenses/by/4.0/

\begin{abstract}
The work is an attempt to find the force with which an electromagnetic system with Foucault currents acts on itself. It is taken into account that the average force with which the source of the alternating magnetic field and the inductive Foucault current is equal to zero, the self-force arises as a result of the interaction of unclosed Foucault conduction currents with the displacement current created by a conductor located in a non-uniform magnetic field. The average force acting on a symmetrical conductor located between the poles of an electromagnet turned out to be different from zero. The greatest value of this force is observed in the region of maximum inhomogeneity of the magnetic field.
\end{abstract}

\section{Keywords}

Eddy Currents, Average Self-Force, Alternative Magnetic Field, Abraham Force

\section{Introduction}

In a massive conductor located in an alternating magnetic field, the so-called Foucault currents arise [1]. They can be induced within nearby stationary conductors by a time-varying magnetic field created by an alternative current electromagnet. The magnitude of the current in a given loop is proportional to the strength of the magnetic field, the area of the loop, and the rate of change of flux, and inversely proportional to the resistivity of the material [2]. Since these currents are inductive, they may not be closed. Something similar happens when an alternator is connected to a capacitor, as shown in Figure 1.

Changing magnetic field $\boldsymbol{B}$ produced by current $I$ will induce an electric field $E$ according to Maxwell's equation: 


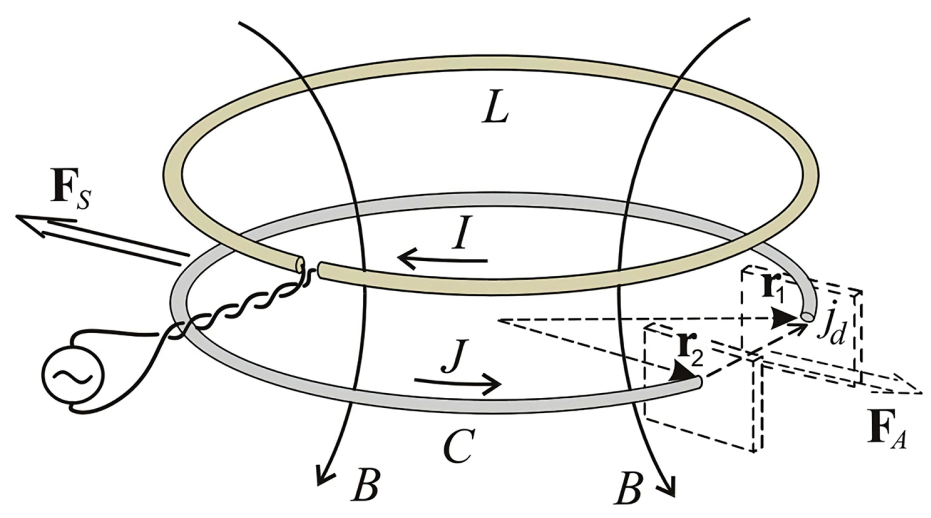

Figure 1. Unclosed conductor in alternative magnetic field.

$$
[\nabla \times \boldsymbol{E}]=-\frac{\partial \boldsymbol{B}}{\partial t}
$$

that means:

$$
J R+\varphi\left(r_{2}\right)-\varphi\left(r_{1}\right)=-\frac{\mathrm{d}}{\mathrm{d} t} \int_{S} \boldsymbol{B} \mathrm{d} \boldsymbol{s}
$$

with integration over surface $S$ bounded by vector $r_{1}-r_{2}$, and the thin conductor $C$ with resistance $R$. In the quasi-stationary approximation, the potential difference $\varphi\left(\boldsymbol{r}_{2}\right)-\varphi\left(\boldsymbol{r}_{1}\right)$, of course, is not equal to zero [3]. The statement about the obligatory closeness of the Foucault conduction currents, based on the neglect of displacement currents, is poorly substantiated. The closure of the total current is provided by the displacement currents that occur between the ends of the conductor [4]. The conduction current in the unclosed part of the conductor $C$ is determined by the time derivative of the magnetic field induction. Since the conduction current is not closed, the self-force:

$$
\boldsymbol{F}_{S}=J\left(\boldsymbol{A}_{C}\left(\boldsymbol{r}_{1}\right)-\boldsymbol{A}_{C}\left(\boldsymbol{r}_{2}\right)\right)
$$

created by the conductor acts on it [5] [6]. Here $\boldsymbol{A}_{C}(\boldsymbol{r})$ is the vector potential of current $J$ at point $\boldsymbol{r}$, of course, proportional to the current $J$. The conservation of momentum is ensured by the force $F_{A}$, formally speaking, acting on the displacement current [7] [8]. Since this force is determined by the square of the current strength, the force averaged over the period of change of the external magnetic field is not equal to zero. Foucault currents in a massive conductor are very large, so the self-force with which an open conductor acts on itself can be significant. A closed conductor, which leads to a closed Foucault current, does not act on itself. That is why the theoretical analysis of the processes occurring with the participation of Foucault currents cannot be considered successful and complete [9]. Displacement currents limit conduction currents. Therefore, without considering the Foucault displacement currents, the theoretical analysis of dynamic processes in conductors located in a non-uniform alternating magnetic field is practically impossible.

If the current in the circuit $L$ changes according to the harmonic law $I=I_{m} \sin \omega t$, 
then the induction of the magnetic field created by the circuit playing the role of an alternating current generator changes in a similar way. The conduction current in the unclosed part of the conductor $C$ is determined by the time derivative of the magnetic field induction. Therefore, the average force over the period $T=$ $2 \pi / \omega$, acting on each current element of the conductor, is equal to zero. The same applies to the average value of the force transmitted to the alternating electromagnetic field, that is, the displacement current. The conduction current flowing in the conductor also acts on the circuit $L$, creating a magnetic force proportional to the product of the current in the conductor $C$ and the current in the circuit $L$. The average value of this force over the period $T$ is also zero. As a result, the entire system consisting of an alternating magnetic field generator and an unclosed circuit must move to start from the electromagnetic field created by this system [10]. This, for example, occurs in a Tamm capacitor [3] [11].

The extremely large value of the current strength is the main feature of the Foucault currents. This means a real possibility of developing a propulsion device that uses an electromagnetic field to create a reactive force. Foucault currents occur at relatively low frequencies [12]. Therefore, in a certain sense, this method of creating an electromagnetic reactive force may turn out to be more promising than EM-drive [13], which requires very high frequencies of an alternating electric field.

\section{System and Its Properties}

Without experimental results, an attempt to propose a way to generate such lift may seem dubious. Shown in Figure 2 a copper disk $C$ with a height $h=4 \mathrm{~mm}$

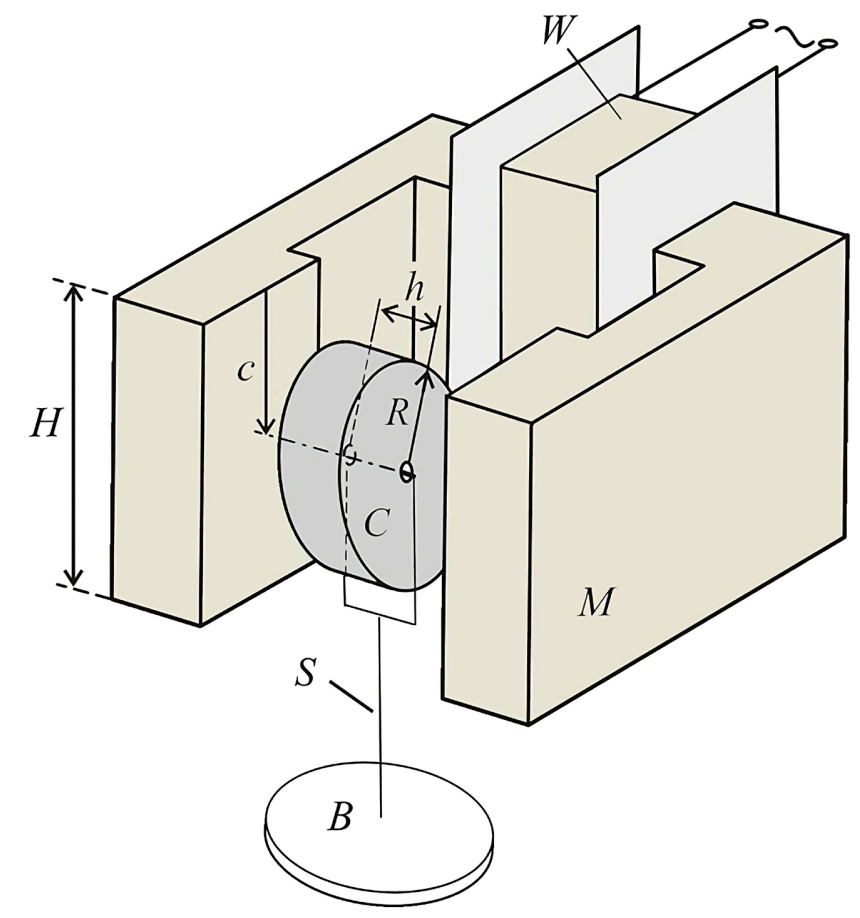

Figure 2. Experimental setup. 
with a radius $R=6 \mathrm{~mm}$ placed between the poles of $\Pi$-shaped core $M$ with alternative current of frequency $50 \mathrm{~Hz}$ plays a role of conductor with Foucault currents. The conductor is connected to a balance $B$ by means of support $S$ made of nonmagnetic materials. The fact that conductor $C$ is symmetrical is of fundamental importance. The force acting on the conduction currents in the conductor, averaged over the period of change of the magnetic field, must be equal to zero, regardless of whether it is in a homogeneous or inhomogeneous magnetic field. The use of an unbalanced conductor would make the interpretation of the experimental results ambiguous [8] [10]. However, the properties of the magnetic field generated by an electromagnet must be described.

First of all, this refers to the determination of the values of $c$, where the magnetic field is uniform. If the conductor is in a uniform magnetic field, there is no preferred direction. In this case, not only the average force, but also its instantaneous value must be equal to zero.

The magnetic field created by the electromagnet is approximately uniform at $04<c / H<0.6$ with $B_{0}=0.052 \mathrm{~T}$ where $H=25 \mathrm{~mm}$, as shown in Figure 3 .

When the magnetic field is turned on, the weight of the system changes significantly (Figure 4). It is still claimed that the weight of the entire system is changing, not just the conductor. Small changes in the position of the conductor relative to the source of the magnetic field lead not only to significant changes in the force acting on the system, but also to a change in its direction.

Even with the position of the conductor close to the region of uniformity of the magnetic field, the weight loss turned out to be very large. Apparently, this is not the limit. One way to find the maximum value of weight loss is to study the dependence of $v$ on the location of the conductor between the poles of the electromagnet.

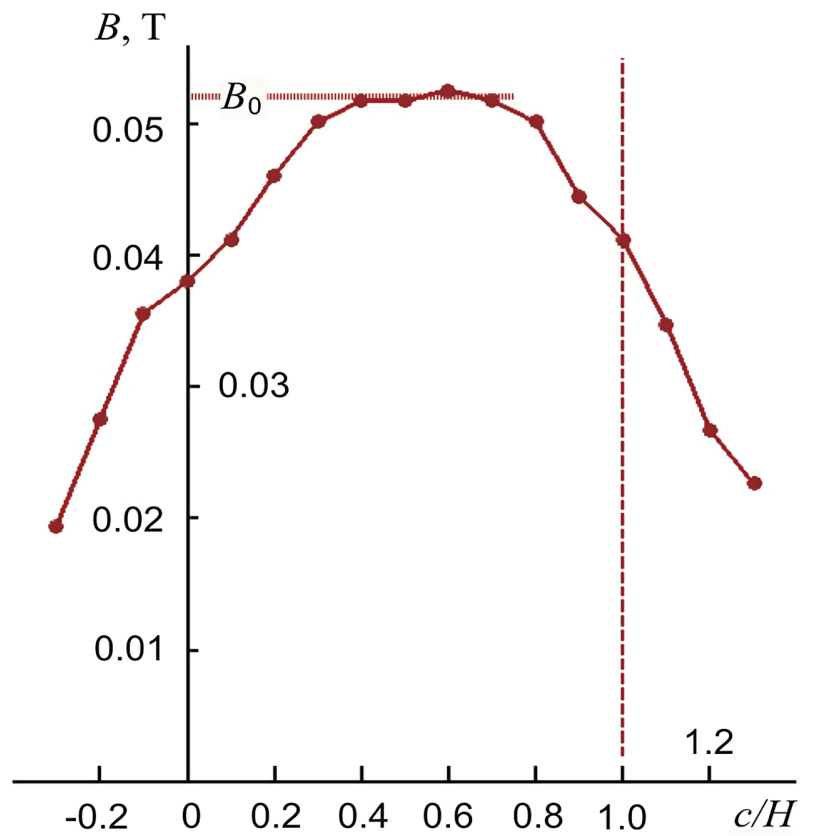

Figure 3. Magnetic field between poles of electromagnet. 


\section{Self-Force and Abraham Force}

There is one more question that should be answered when studying the dependence of $\Delta P$ on $c$. this dependence must be antisymmetric with respect to $c=$ $H / 2$. In other words, the values of $\Delta P$ at $c=0$ and at $c=H$ should differ only in sign if the interaction of the Foucault currents in the conductor with the environment, including laboratory equipment, is negligible. Otherwise, the statements that the values of weight change $\Delta P$ represent the reaction force of electromagnetic

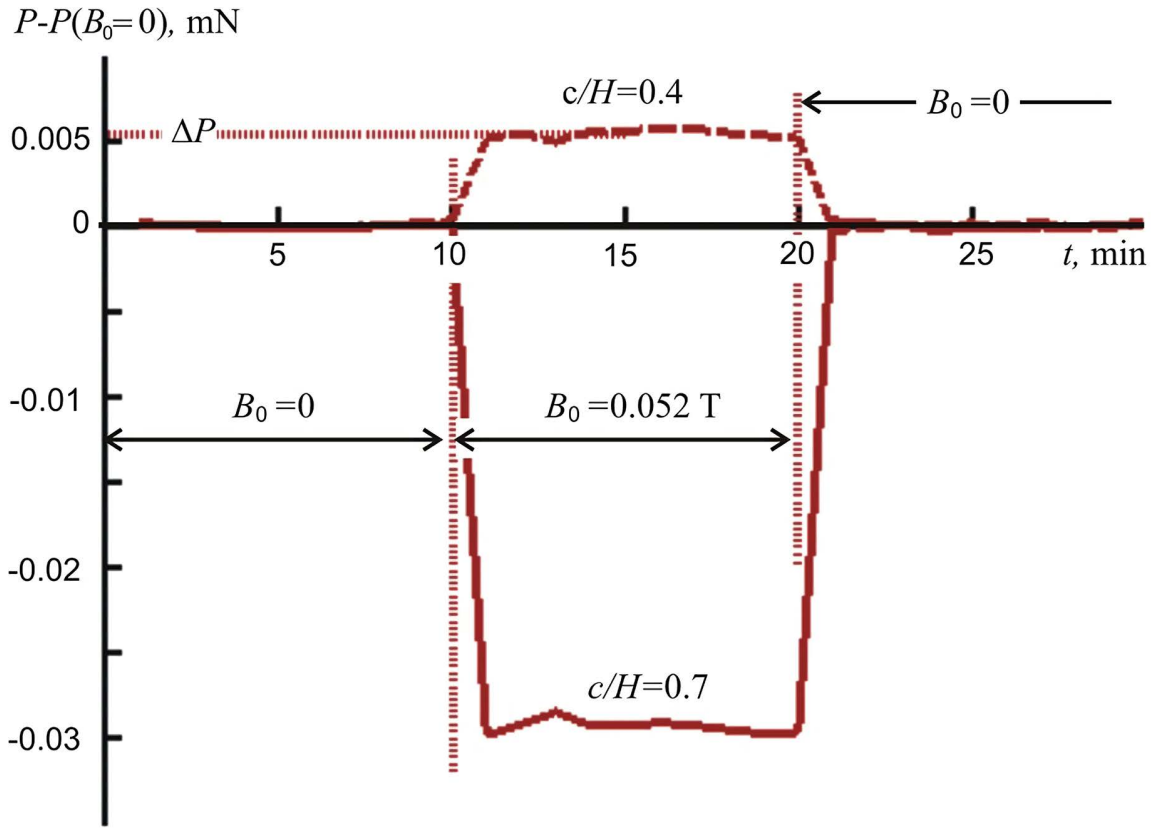

Figure 4. Weight of the system at turning the magnetic field.

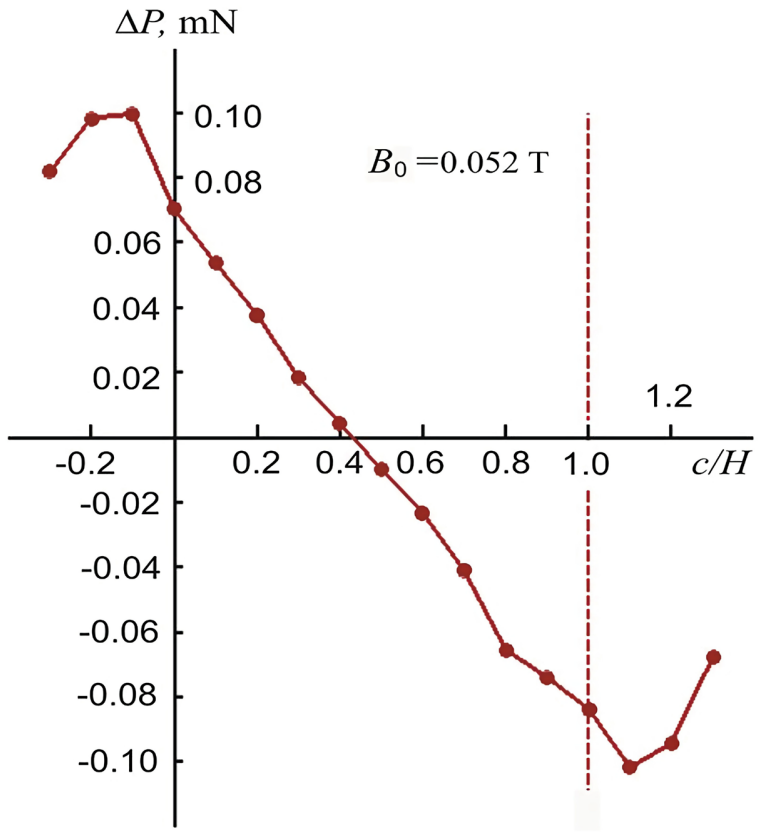

Figure 5. Force acting on conductor at various positions. 


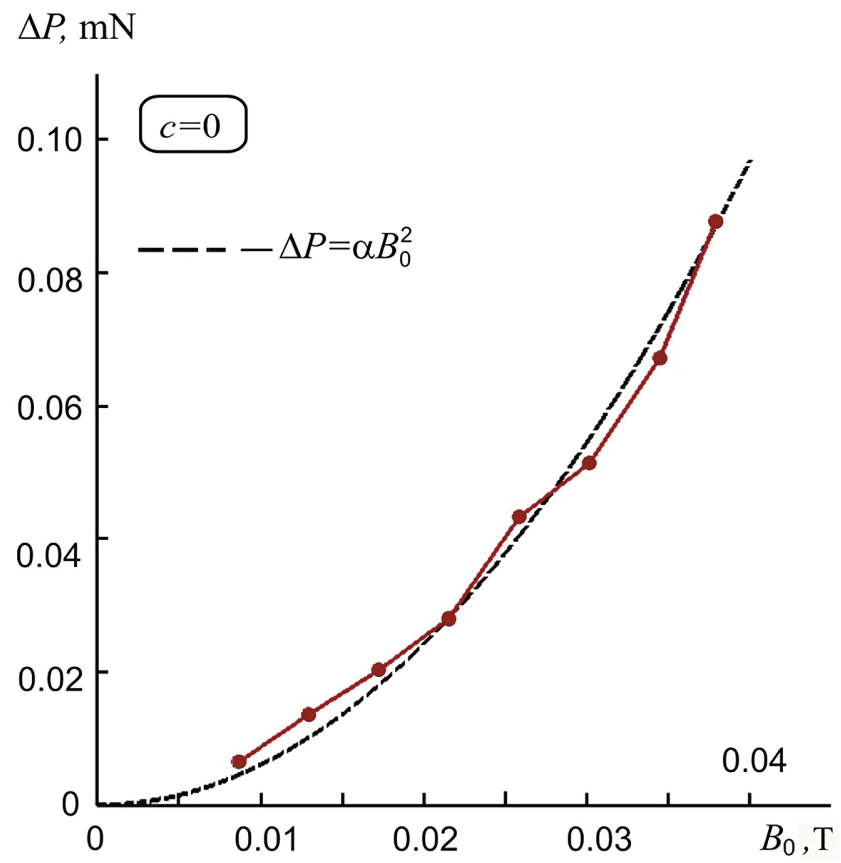

Figure 6. Influence of magnetic field inductance on weight of conductor.

radiation created by the conductor and acting on the conductor seem doubtful. In the first approximation, the dependence of the weight change on the distance $c$ turned out to be antisymmetric (Figure 3 ). In this case, the maximum deficit or surplus is observed when the center of the conductor is located on the boundary of the electromagnet cavity, where the magnetic field is most inhomogeneous. This is shown in Figure 5.

If the weight loss is due to interaction with the environment, then the dependence of $\Delta P$ on the magnetic field inductance should be linear. If this is the result of self-action, then the change in weight must be proportional to the square of the induction of the magnetic field created by the source. Dependence $\Delta P$ on $B_{0}$ (Figure 6) shows that the dominant role in the system shown in Figure 1 is the self-action described by Abraham's force.

\section{Conclusion}

The Foucault current density is proportional to the frequency of magnetic field oscillations. The above results correspond to a frequency of $50 \mathrm{~Hz}$. Increasing the frequency to $5 \mathrm{kHz}$ leads to a hundredfold increase in the Foucault current density. An increase in the induction of an inhomogeneous magnetic field $B_{0}$ leads to a hundredfold increase in the Abraham force. This can be achieved, among other things, by changing the shape of the electromagnet. Therefore, after such changes, the maximum weight loss can reach $1 \mathrm{~N}$. This is only for one cell. The systems are shown in Figure 2, remote from each other by a distance of the order of $H$, there can be a lot. There are other ways to increase the power of Abraham. Changes in the size and shape of the conductor can also affect the magnitude of this force, but so far remain outside the scope of this work. Enough has been said to draw at- 
tention to this mode of electromagnetic propulsion.

\section{Conflicts of Interest}

The author declares no conflicts of interest regarding the publication of this paper.

\section{References}

[1] Wangsness, R.K. (1986) Electromagnetic Fields. John Wiley \& Sons, New York.

[2] Fiorillo, F. (2004) Chapter 7-Characterization of Soft Magnetic Materials. In: Measurement and Characterization of Magnetic Materials, Elsevier Academic Press, Amsterdam, 307-474. https://doi.org/10.1016/B978-012257251-7/50009-5

[3] Tamm, I.E. (1979) Fundamentals of the Theory of Electricity, Mir Publisher, Moscow.

[4] Gerasimov, S.A. (2019) Reactive Electrodynamics of Unclosed Conductor with Alternative Current. Engineering Physics, 19, 35-38.

[5] Cavalleri, G., Bettoni, G., Tonni, E. and Spavieri G. (1978) Experimental Proof of Standard Electrodynamics of Measuring the Self-Force on a Part of Current Loop. Physical Review E, 58, 2505-2517. https://doi.org/10.1103/PhysRevE.58.2505

[6] Gerasimov, S.A. (2014) Interaction of Current Elements, Conservation of Momentum and Pseudo-Self-Action in Magnetostatics. Applied Physics and Mathematics, 3, 3-6.

[7] Gerasimov, S.A. (2021) On Reactive Force in Thin Unclosed Conductor and Displacement Current. European Journal of Applied Physics, 3, 7-10.

https://doi.org/10.24018/ejphysics.2021.3.5.100

[8] Gerasimov, S.A. (2021) Dynamics of a Conductor with Unclosed Alternative Electric Current. Russian Electromechanics, 64, 12-15.

https://doi.org/10.17213/0136-3360-2021-6-12-15

[9] Rodriguez, F.F. and Valli, A. (2010) Eddy Current Approximation of Maxwell Equation: Theory, Algorithms and Applications, Springer-Verlag, Mailand. https://doi.org/10.1007/978-88-470-1506-7 9

[10] Gerasimov, S.A. (2021) Foucault Currents in Unclosed Conductor and Abraham's Force. Engineering Physics, 21, 37-40.

[11] Graham, G.M. and Lahoz, D.G. (1980) Observation of Static Electromagnetic Angular Momentum in Vacuo. Nature, 285, 154-155. https://doi.org/10.1038/285154a0

[12] Gerasimov, S.A. (2020) Two Letters about Reactive Electrodynamics and Electromagnetic Antigravity. IOSR Journal of Applied Physics, 12, 1-4.

[13] Wu, C.W. (2018) Comments on Theoretical Foundation of "EM Drive". Acta Astronautica, 144, 214-215. https://doi.org/10.1016/j.actaastro.2018.01.006 